\title{
El diagnóstico y tratamiento de las dislexias ante un modelo de lectura normal
}

\author{
LUIS LOZANO \\ Equipo Interdisciplinar del Sector de Avilés
}

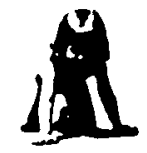

Resumen

El objetivo fundamental de este trabajo es triple. Por una parte se expone el marco general que se asune. El modelo de lectura de doble rita, encuadrado en los trabajos sobre el Procesamiento de la Información. propone una arquitectura funcional determinada por un nímero determinado de módulos con unas especificas comunicaciones entre ellos. A partir de aquí, conjuntamente con los datos experimentales que se conocen sobre cómo funciona la lectura en los buenos lectores, se utiliza para elaborar un diagnóstico de las dificultades Lectoras. Este diagnóstico buscará el conociniento de aquellas babilidades especificas que tienen que ver con la lectura y que por diversas circunstancias no funcionan adecuadamente. Por último, se planifica el tratamiento específico dirigido exclusivamente a los aspectos pertienentes a la lectura, sin divagar sobre otros aspectos clásicos (psicomotricidad, lateralidad, etc.) que nada tienen que ver con la lectura.

Palabras clave:Dislexia superficial; Dislexia fonológica; Ruta fonologica: Ruta léxica; Modelo de lectura de doble Ruta

\section{Diagnosis and Treatment of Dyslexia Based on a Normal Reading Model}

\section{Abstract}

The aims of this study are threefold. First, it explains the study's general model: The double route reading model from an information processing perspective. It proposes a functional arcbitecture determined by a specific number of components with precise communication between them. Secondly, this model, togetber with the available experimental data on expert reading, is used to diagnose reading difficulties. This diagnosis looks for the specific skills involved in reading which for different reasons are not being used adequately. Finally, the subject's specific treatment is elaborated. This is directed exclusively to relevant aspects of the reading process, without digressing onto other classical aspects (psychomotive, laterality, etc.) which are unrelated to the reading process.

Key words: Surface dyslexia; Pbonological dyslexia; Pbonological route; Lexical route; Double route reading model. 


\section{INTRODUCCION}

Muchas investigaciones y taxonomías que tratan la lectura y sus problemas se caracterizan por estar centradas en los niños que leen mal, en las habilidades que estos niños tienen o no adquiridas y que son consideradas, por tanto, como la causa que origina las dificultades lectoras, lo cual puede traer consigo discutibles enfoques correlacionales. Este trabajo ha dejado de lado esta orientación y centra los esfuerzos en la descripción de las características cualitativas y cuantitativas de la lectura de los sujetos.

Ahora bien, para que estos datos puedan interpretarse y compararse sistemáticamente de la manera más eficaz posible es necesario un marco general como el suministrado por los modelos de lectura normal. Sólo desde el conocimiento de lo que sucede en la lectura normal podemos organizar el proceso de enseñanza-aprendizaje de la lectura en las aulas ordinarias con codos los sujetos. Se lograría así un cuerpo teórico que, además de orientar la práctica educativa sobre una base experimental y no sobre mitos psicopedagógicos, serviría para explicar por qué ocurren determinadas dificultades lectoras en algunos niños. Es evidente que si se logra saber la causa de estas dificultades, tendremos mucho camino andado para elaborar las actividades terapéuticas adecuadas dirigidas a la superación de las mismas.

El marco teórico que se asume en este trabajo es el que suministra la teoría del Procesamiento de la Información. Se considera que la lectura es producto de una transformación de la información de una forma de representación a otra, asumiendo que cada una de estas transformaciones se realiza por un componente específico del modelo de lectura propuesto. Así pues, el sistema de lectura lo consideramos formado por un número de componentes o módulos independientes entre sí, cada uno responsable de la transformación de un tipo de información a otro y con una clara comunicación entre ellos. Desde la teoría de la doble ruta (Figura 1), se propone la existencia de dos formas de leer las palabras: un procedimiento léxico y otro procedimiento no léxico (Morton, 1969, 1980; Coltheart, 1978, 1980; Morton y Patterson, 1980; Marshall, 1984, 1985, etcétera).

Cualquiera de estas dos rutas permite reconocer o leer la palabra y el uso de una u otra ruta vendrá determinado por la velocidad relativa de cada una y/o por la estrategia concreta que adopte el sujeto.

La ruta de procesamiento léxico o visual se caracteriza porque sólo funciona con las palabras que forman parte del vocabulario visual u ortográfico del sujeto. Evidentemente, las palabras desconocidas por el sujeto, las no palabras y las pseudopalabras no se pueden leer por esta ruta al no tener su correspondiente representación léxica. Como de hecho somos capaces de leer estas tres clases de estímulos debe existir otra ruta por la cual se les pueda dar una respuesta: es la ruta de procesamiento no-léxico o fonológico.

Debemos tener en cuenta que cada una de estas rutas está formada por diversos subprocesos que es necesario describir al objeto de comprender de forma correcta cómo se produce la lectura normal. Es así que la ruta de procesamiento léxico, como se observa en la Figura 1, está compuesta fundamentalmente por tres léxicos diferentes:

El léxico ortográfico (Reconocimiento de las Palabras), formado por todas las palabras que el sujeto conoce visualmente, es el encargado de determinar si el estímulo visual es o no una palabra del idioma. Una vez reconocida la palabra se puede acceder o no a su significado en el Sistema Semántico (Comprensión de Palabras). Este sistema es común para todas las funciones de comprensión y expresión del suje- 
to, sean escritas, habladas, pictográficas, etc. El tercer elemento es el Léxico Fonológico (Pronunciación de Palabras), donde se encuentran todas las palabras que el sujeto conoce por sus sonidos, por su pronunciación. A este léxico puede llegarle la información proveniente del Sistema Semántico (el significado de la palabra), a partir del cual se logra su pronunciación permitiendo, en último término, la lectura en voz alta. Se asume que esta trayectoria desde el Sistema Semántico al Léxico Fonológico es la que se sigue en el discurso espontáneo que convierte las ideas que deseamos transmitir en el lenguaje hablado (Coltheart, 1984). Ahora bien, como estos tres subprocesos son independientes entre sí, existe de hecho otra comunicación entre el Reconocimiento de las Palabras y la Pronunciación de Palabras sin pasar por la Comprensión de Palabras. Utilizando esta vía, evidentemente no se puede comprender el significado de la palabra, pero es necesario contemplarla ya que existen datos de sujetos que siendo incapaces de leer pseudopalabras, pueden leer las palabras con una comprensión nula (Schawartz, Saffran y Marin, 1980; Funell, 1983; Aram, Rose y Horwitz, 1982; Silberberg y Silberberg, 1967).

Por lo dicho, a través de esta ruta léxica se pueden leer todas las palabras (regulares o irregulares), pero no las pseudopalabras, pues de leerlas por esta ruta se producirían lexicalizaciones. Es decir, ante una pseudopalabra se respondería con una palabra, ya que es el único tipo de respuesta que esta ruta puede realizar. Además es importante recordar que la frecuencia de uso es una variable significativa en el reconocimiento de las palabras en esta ruta.

FIGURA 1

Modelo de lectura de Coltheart (1981)

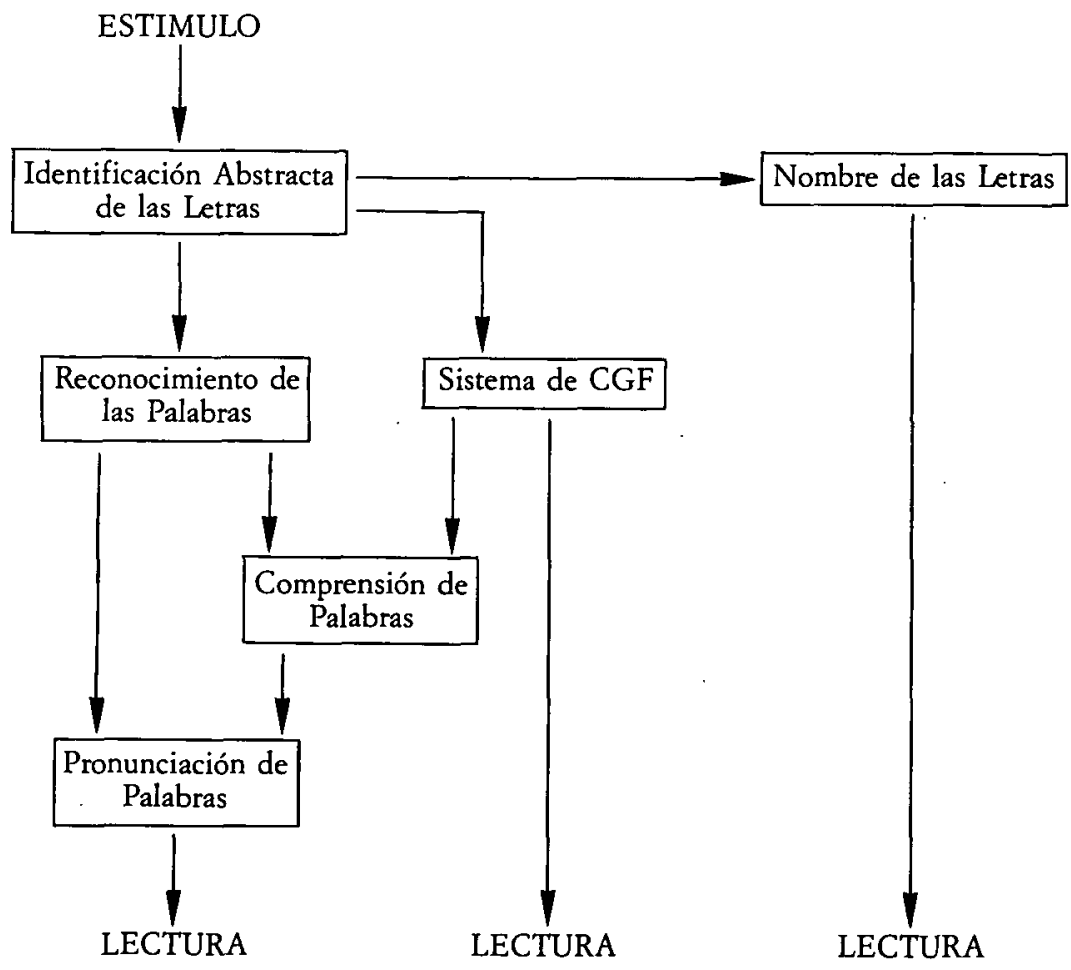


En la otra ruta de procesamiento no léxico o fonológico la unidad de analisis ya no es la palabra en cuanto tal, sino que el conocimiento representado es el conocimiento de las reglas abstractas de Conversión Grafema a Fonema (CGF). Este proceso está formado por tres mecanismos. Cuando a un sujeto se le presenta una palabra o una pseudopalabra ha de realiar un análisis grafémico con el objeto de identificar unidades más pequeñas tales como el grafema. Una vez identificados éstos se asignaría a cada grafema el fonema correspondiente, para en un tercer momento ensamblarlos al objeto de pronunciarlos todos conjuntamente tal y como hablamos normalmente (Coltheart, 1978, 1984).

En esta ruta es evidente que se puede producir una pronunciación correcta de cualquier pseudopalabra y de las palabras regulares. Si la palabra fuese irregular, al adjudicar a cada grafema el fonema más común se producirían las regularizaciones, de aquí que en los idiomas opacos como el inglés o el francés sea necesaria la ruta léxica, pues de no ser así no se podría leer la enorme cantidad de palabras que tienen una única y específica pronunciación. En esta ruta la frecuencia no es la variable pertinente, pues tanto las palabras de frecuencia alta como las de frecuencia baja han de sufrir idénticos procesos. Sin embargo, se considera que la longitud es la variable que afecta al rendimiento lector, pues cuanto más larga sea la palabra más traducciones de grafemas habrá que hacer y, en consecuencia, la probabilidad de que se produzca un error será mayor (para un análisis más detallado de los modelos de lectura se puede consultar en castellano a Valle, 1991; Cuetos, 1990; Cuetos y Valle, 1990; Loano, 1990, 1991).

Este modelo de lectura hace hincapié en el hecho de que un buen lector ha de ser capaz de utilizar los procedimientos implicados en ambas rutas. En consecuencia, asumir un modelo de estas características trae importantes consecuencias no sólo en cómo hay que enseñar a leer, sino en cómo enfocar el análisis de las dificultades lectoras. Desde esta perspectiva, asumimos que las dislexias evolutivas han de interpretarse como consecuencia de un fracaso en el funcionamiento de algunos de los módulos o de las comunicaciones que entre ellos existen (Marshall, 1984, 1985; Coltheart, 1981, 1984).

La oportunidad de poner en práctica, sobre la realidad de la escuela, los postulados teóricos defendidos en este trabajo y comprobar si se muestran eficaces en la elaboración del diagnóstico y del tratamiento nos la brindó el caso que se expone a continuación y que llegó a consulta después de un verdadero peregrinaje por distintos servicios médicos y psicopedagógicos.

\section{ESTUDIO DE UN CASO DE DISLEXIA EVOLUTIVA}

\section{Proceso de valoración}

El nombre del alumno es Iván y nació el 8 de diciembre de 1978. La intervención se realiza a petición de una autoridad educativa al objeto de comprobar si es necesario, dadas las dificultades escolares que presenta, reorientar su escolarización hacia un Centro de Integración. Se contacta con la familia y el niño en octubre de 1989.

Cuando llegó a consulta en octubre de 1989, con 10 años y 10 meses, ya presentaba una historia amplia de informes y valoraciones. En julio de 1987 se le detectó en la Unidad de Foniatría de la Ciudad Sanitaria importantes trastornos de lecroescritura, carencia de agilidad articulatoria, múltiples dislalias, ligera rinofonía y una discreta disfonía, aconsejando simplemente rehabilitación logopédica. 
En febrero de 1988 , estando en $3 .^{\circ}$ de EGB, se le realiza un informe psicopedagógico donde lo más característico es que presenta una capacidad intelectual normal (WISC, CIt $=96, \mathrm{CIv}=85, \mathrm{CIm}=107$ ). En el Bender únicamente se hace referencia a una puntuación dentro de la normalidad, sin indicar si existe algún tipo de indicadores emocionales o neurológicos. En el TALE se le detectan problemas de silabeo, inexactitud lectora y distorsión de la lectura en algunas palabras, mientras que en la escritura muestra inversión de sílabas (convierte las directas en inversas), sustituye unas vocales por otras y no separa adecuadamente las palabras de la frase. También se le recomienda rehabilitación logopédica.

En octubre de 1988 se le aprecian grandes avances en la pronunciación, persistiendo la dificultad en el fonema $/ \mathrm{r} / \mathrm{y}$ los problemas de lectoescritura.

Iván es el menor de tres hermanos. Los antecedentes familiares que destacan son: su padre y hermano tardaron en hablar, dos primos sordos por meningitis, una hermana taquifémica y problemas de aprendizaje en sus dos hermanos.

Sus antecedentes personales que constan son: embarazo y parto normales, desarrollo psicomotor normal y un desarrollo del lenguaje normal en cuanto a su evolución sintáctica y pragmática, no así en los aspectos fonológicos del habla. Realizó rehabilitación logopédica durante 4 años. Se adapta bien al colegio, repitió $1 .^{\circ}$ de EGB por bajos rendimientos en el área del lenguaje. Cuando llegó a consulta estaba escolarizado en 5. de EGB. Acude al aula de Apoyo en el colegio y a clase particular una hora diaria. En casa la madre estudia con él, lo que le produce una insistencia materna agobiante.

Actualmente mentiene una conversación tanto a nivel comprensivo como expresivo acorde a su edad cronológica.

\section{Valoración Psicopedagógica}

La actitud que muestra Iván es de absoluta y franca colaboración. Se le pasa la Escala de Alexander obteniendo una puntuación equivalente a 12 años y medio en Passalong, lo que unido a los datos del WISC descartan cualquier tipo de retraso intelectual.

En la Prueba de ITPA muestra un retraso general importante (obtiene una EPL = 7 años y medio), aunque lo que destaca específicamente sobre su media global de 32 (dentro totalmente de la normalidad) son, tomando como criterio 6 puntuaciones típicas, las siguientes subpruebas: Comprensión visual (47) y Expresión motora (39) por la parte alta y una importante dificultad en Asociación Auditiva (23) y Memoria secuencial auditiva (22). Este último dato fue reafirmado por la prueba de Memoria Auditiva Inmediata (MAI), donde sólo alcanzó un percentil entre 5 y 10 . Estos resultados nos hacen hipotetizar que presenta superioridad del canal visomotor sobre el auditivo vocal, observándose algunas dificultades en los procesos receptivos (los expresivos se fueron corrigiendo progresivamente) y una clara diferencia a favor de los niveles representativos frente a los automáticos.

Dado que las dificultades más constantes a lo largo de su amplia historia eran los problemas de lectura, se decidió efectuar un estudio sistemático de los errores que cometía.

El objetivo que nos propusimos, en primer lugar, fue cambiar el centro de atencion que desde siempre se tuvo con Iván. Es decir, había que centrarse en cómo lee, más que en las características del propio Iván, de forma que nos permitiera hacernos una idea de qué es lo que ocurre cuando se pone a leer. Para ello se le grabó la lectura 
de 144 palabras controladas por su longitud, frecuencia y clase gramatical. La razón estaba en que deseábamos conocer las características cualitativas y cuantitativas de su lectura para, una vez comparada con las que muestran los alumnos de $1 . .^{\circ}, 3 .^{\circ}$ y $6 .^{\circ}$ de EGB, hipotetizar qué aspectos o habilidades del modelo están fallando y plantear la terapia que se considere oportuna dependiendo de los resultados obtenidos.

Los resultados de este análisis han-sido los siguientes:

Cometió significativamente mienos errores en la lectura de las palabras que en las pseudopalabras ( $58 \%$ frente a un $88,11 \% ; Z=3,743>2,58, p<0.01$ ). Observando la relación de errores NP/P (número de errores en las pseudopalabras divididos entre el número de errores en las palabras), nos da 1,47 , que es menor incluso que la mostrada por los alumnos de $1 .^{\circ}(1,52)$. Es decir, los alumnos de $1 .^{\circ}$ leen proporcionalmente mejor las palabras que Iván, tanto en términos relativos como absolutos. La media de errores en $1 .^{\circ}$ en las palabras era de 11,55 y 17,58 en las pseudopalabras, mientras que Iván comete 42 y 62 , respectivamente. Sin embargo, el comportamiento general de Iván ante las palabras de FA $(28,57 \%$ del total de los 42 errores cometidos) en comparación con las de FM $(40,47 \%)$ y FB $(30,95 \%)$ es parecido al que muestran los sujetos de $1 .^{\circ}$, aunque como hemos dicho, más agravado en términos absolutos. Si comparamos el $28,57 \%$ de los errores en las palabras de FA con la media de errores en las palabras de FM y FB $(35,7.1 \%)$ las diferencias son significativas $(Z=0,607>-1,96, p 0,05)$ al igual que sucede en los sujetos de $1 .^{\circ}$. Estos se comportan significativa y diferencialmente ante las palabras de FA frente a las de FM y FB. Todos estos resultados nos permiten indicar que Iván presenta una lectura equivalente al grupo de $1 .^{\circ}$, es decir, 5 años de retraso con respecto a su edad cronológica, lo cual es un indicio de lo inhabilitada que tiene su lectura por la ruta léxica.

Si observamos el efecto que puede ejercer la longitud en la lectura de las palabras, se encuentran unos datos ciertamente significativos. De los 42 errores en las palabras, 25 lo son en las palabras de LL, 10 en las de LM y 7 en las de LC. Por otra parte, de los 62 errores que realiza en las pseudopalabras 33 son en las de $\mathrm{LL}, 17$ en las de LM y 12 en las de LC. Efectuando el recuento global el 55,76\% de los errores lo son en los estímulos de LL, el 25,96\% en las de LM y el 18,26\% en las de LC. Estos datos muestran claramente un efecto de la longitud en la lectura de los 144 estímulos, cometiendo un $10 \%$ de errores más en las palabras de LL que los sujetos de $3 .^{\circ}$ y de $1 .^{\circ}$.

Contabilizando las lexicalizaciones, producidas por un funcionamiento de la ruta léxica, se encontraron 10 , equivalentes a un $16,12 \%$ de los errores en las pseudopalabras.

Puede observarse en la Tabla I que todas las lexicalizaciones, excepto una, conservan los primeros o últimos fonemas, lo que equivaldría, en opinión de Stuart y Coltheart (1988), a un efecto eminentemente fonológico. Por tanto, si al efecto léxico le restásemos aquél, que puede ser producido por el efecto fonológico, se quedaría en $1,61 \%$, ya que sólo una pseudopalabra no cumple el que se mantengan los primeros o últimos fonemas en la respuesta lexicalizada. Luego el efecto de la ruta léxica en Iván es muy pequeño y más en comparación con los alumnos de $1 .^{\circ}$ de EGB, cuyas respuestas lexicalizadas han sido un $24,91 \%$ que se reduciría a un $16,83 \%$ después de descontar las producidas por el efecto fonológico.

El efecto complementario a las lexicalizaciones, la lectura de las palabras como pseudopalabras, producto de un funcionamiento de la ruta fonológica, es mucho mayor incluso que en $1 .^{\circ}$. De la 42 palabras que leyó erróneamente, 35 (el 83,33\%) las convirtió en pseudopalabras, no observando ningún efecto de frecuencia. Así, 
convirtió en pseudopalabras 12 de FA, 13 de FM y 10 de FB. Sin embargo, se encontró un efecto muy claro de la longitud, ya que de las 35 palabras convertidas en pseudopalabras, 25 eran de LL, 7 de LM y 3 de LC, lo que indica claramente una lectura a través de las reglas de CGF.

Si consideramos como error visual aquella lectura que contiene al menos el $50 \%$ de las letras de la palabra y el mantenimiento de cierto orden secuencial, Iván cometió únicamente 3 errores visuales $(7,14 \%)$. Así, leyó «completar" por "contemplar» y «desayuno" por "desayunar». A hora bien, en las pseudopalabras produjo un $60,51 \%$ de pseudopalabras con parecido visual (leía «vandadero» por «vardadero», «anteresarto" por «anteresante», "siquiero» por «siquiera», etc.) y un 9,6\% de palabras, también con parecido visual (ver las lexicalizaciones). Las omisiones representaron el $12,9 \%$.

Cometió una sustitución semántica al leer «gallo» ante «pollo» que, dadas las características de la lectura de Iván, se consideró más un error visual que de sustitución semántica.

La comprensión de lo que lee se obtiene a partir de la fonología final. Así se obtuvieron las respuestas de la Tabla II. Las palabras a la izquierda de la flecha son el estímulo y a continuación se exponen las contestaciones realizadas y entre paréntesis el significado que dio a lo que ha leído, finalizando entre interrogaciones con aquello que nosotros pensamos que, por su definición, ha entendido.

TABLA I

Lexicalizaciones

\begin{tabular}{|c|c|c|}
\hline & $\begin{array}{c}\text { ESTIMULO } \\
\text { sanvo } \\
\text { entrecanto } \\
\text { recamendar } \\
\text { preserciar } \\
\text { turso } \\
\text { herramienla } \\
\text { venfa } \\
\text { encapaz } \\
\text { socesivamente } \\
\text { glave }\end{array}$ & $\begin{array}{l}\text { LECTURA } \\
\text { salva } \\
\text { encanto } \\
\text { recornendar } \\
\text { presenciar } \\
\text { puso } \\
\text { ramillete } \\
\text { rifa } \\
\text { en capas } \\
\text { socialmente } \\
\text { gr ave }\end{array}$ \\
\hline \multicolumn{3}{|c|}{$\begin{array}{c}\text { TABLA II } \\
\text { Proceso en la búsqueda de comprensión }\end{array}$} \\
\hline «cabalmente" & \multicolumn{2}{|c|}{$\rightarrow$ «cabalmente" $\rightarrow$ "calvamente" (que tiene calva). } \\
\hline «diverso" & \multicolumn{2}{|c|}{$\rightarrow$ «birverso» (alguien que es muy malo) ¿perverso? } \\
\hline «transparencia» & \multicolumn{2}{|c|}{$\rightarrow$ «trantarencia» (que alguien se comunica) ¿conferencia? } \\
\hline «por tanto" & \multicolumn{2}{|c|}{$\rightarrow$ «protanto» (que va rápido) ¿trotando? } \\
\hline «importante» & \multicolumn{2}{|c|}{$\rightarrow$ «repartiente» (que reparte alto) ¿repartidor? } \\
\hline «obsarvación» & \multicolumn{2}{|c|}{$\begin{array}{l}\rightarrow \text { «osarvación» (que un señor salva a alguien que est-a en un pozo o } \\
\text { algo así) ¿salvación/salvamento? }\end{array}$} \\
\hline «bediante» & \multicolumn{2}{|c|}{$\cdot \rightarrow$ «debinante» $\rightarrow$ «grinate» $\rightarrow$ «debiante» $\rightarrow$ «brillante» (que brilla) } \\
\hline
\end{tabular}


Fijándonos en aspectos menos cuantitativos verificamos que subvocaliza antes de dar la lectura completa, quizá debido a la estrategia que le proporcionaron los maestros y logopedas para que no silabee. Gracias a esta estrategia se obtuvieron bastantes datos sobre cómo funciona en la conversión de los grafemas a fonemas. Se observó que analiza incorrectamente la palabra en sus grafemas constituyentes. Es así que ante "inagotable» lee «in/na/go/ta/ble», ante "patrionismo» hace "pe/tri/en/is/mo», ante «excesivo» lee «ex/ces/si/vo».

Por otra parte, no asocia bien los fonemas a los grafemas correspondiente, pues, además de los ejemplos ya mencionados, ante «preger» leyó «pregal», ante «conferencia» leyó «conterencia», ante «escondito» leyó «seconbito», etc., aunque cuando se le mandaba que deletrease las palabras lo realizaba correctamente.

También se encontró, a partir de la subvocalización, una importante dificultad en el intento de ensamblar los fonemas correctamente logrados que corresponden a los grafemas. Iván cuando comienza a leer (ver la Tabla III) va desgranando las sílabas y cuando termina mira hacia arriba, al techo, e intenta ensamblar la fonología obtenida.

TABLA III

Ensamble de silabas

$\begin{array}{lll}\text { «calvamente» } & \rightarrow \text { «ca-bal-men-te» } & \rightarrow \text { «cabalmente» } \\ \text { «obsarvacion» } & \rightarrow \text { «o-bes-va-ci-on» } & \rightarrow \text { uosarvación» } \\ \text { «mucroscopio } & \rightarrow \text { «mu-cres-ca-pi-o» } & \rightarrow \text { «escapio» } \\ \text { «inagotable> } & \rightarrow \text { «in-na-go-ta-ble» } & \rightarrow \text { «ingratraba» } \\ \text { «sentimiento» } & \rightarrow \text { «sen-ti-mien-to» } & \rightarrow \text { «sentiendo» }\end{array}$

Teniendo presentes las dificultades que su historia clínica tenía en cuanto a las dificultades articulatorias se intentó descartar que los problemas de lectura estuvieran causados por este aspecto, por lo que se le fue diciendo oralmente las palabras que leía mal. El resultado fue que casi todas las repitió correctamente en el primer intento, sólo 7 las repitió mal, pero al decirle que estaban mal las corrigió espontáneamente.

Se comprobó que era capaz de segmentar oralmente en sílabas las palabras habladas, quitar y añadir una sílaba, quitar y añadir un fonema, comparar sonidos al principio, en medio y al final de dos palabras. Es decir, los prerrequisitos de la lectura en cuanto a segmentación fonológica y conciencia fonológica (Alegría, 1985; Sebastián y Maldonado, 1984), además del conocimiento de las letras, estaban logrados de forma adecuada.

\section{Conclusiōn}

El primer comentario que hay que hacer es poner de manifiesto, una vez más, la importancia que tiene la lectura en el desarrollo escolar, hasta el punto de que un niño con una capacidad intelectual normal al igual que en las demás habilidades escolares, está a punto de ser considerado sujeto de integración. Es decir, sujeto minusválido, por no ser capaz de leer adecuadamente según el criterio que la escuela define y determina para su edad y curso escolar. 
Los datos aquí mostrados indican que Iván prensenta unas características lectoras típicas de una dislexia fonológica, en similitud a la que presentan los sujetos adultos con dislexia fonológica adquirida (Valle, 1991; Cuetos, 1990; Lozano, 1990, 1991).

Por una parte, Iván no tiene problemas en los análisis visuales previos, como se demuestra por su rendimiento en las pruebas estandarizadas como el Bender, el Passalong, el mejor funcionamiento en el área visomotora del ITPA, además de la buena capacidad de deletrear las letras de izquierda a derecha de las palabras o pseudopalabras que lee defectuosamente, lo cual nos permite descartar que sus dificultades sean periféricas, es decir, sean producto de análisis visuales previos al procesamiento semántico o fonológico de las palabras.

Sin embargo, Iván muestra ciertas peculiaridades. Presenta dificultades en el análisis grafémico, pero la mayor dificultad se encuentra en la asociación de los fonemas con los grafemas correspondientes y en el ensamblaje de los fonemas logrados sean o no correctos. Es decir, el módulo de CGF no funciona adecuadamente en ninguno de sus tres subprocesos. Son dificultades propias de la ruta no léxica.

Por otra parte, la ruta léxica tampoco está funcionando correctamente, como ya se ha expuesto. Se mantiene que es el módulo de Reconocimiento de las Palabras el que no funciona adecuadamente, como nos lo indica el $58,33 \%$ de errores en la lectura de palabras.

De forma totalmente coherente con este aspecto hay que tener presente que la comprensión de lo leído no se hace a través de la ruta léxica, sino a partir de la fonología obtenida por la ruta no léxica. De ahí los errores en su comprensión de lo escrito, ya que tampoco la ruta fonológica produce fielmente el sonido de la palabra. De hecho, una estrategia escolar es grabarle las lecciones en cintas magnetofónicas para que a partir del lenguaje oral pueda adquirir los conocimientos que le están vedados si utiliza el lenguaje escriro.

Otro aspecto interesante a destacar es lo clarificador que resulta comparar los datos obtenidos en la lectura individual de palabras de Iván con los obtenidos por una muestra poblacional. De esta forma nos permite describir su lectura en términos más cualitativos y cuantitativos, pudiendo establecer distintas hipótesis sobre qué está sucediendo en su lectura, como aquí se ha hecho.

\section{TRATAMIENTO}

Dado que había que aprovechar las posibilidades integradoras que el aula tiene, el tratamiento no se planificó con una idea exclusivamente experimental, con lo que hubo aspectos que, evidentemente, no se pudieron controlar y otros seguramente no son pertinentes, en sentido estricto, con la orientación teórica defendida. Se mantuvieron reuniones con las tres profesoras (tutora, de apoyo y particular) y con la madre (fue imposible reducir el número de personas intervinientes en el caso), donde se planteó la estrategia terapéutica. Los objetivos generales eran los siguientes:

1. Elevar su autoestima personal en lo referente a su capacidad para afrontar las tareas de lectura, ya que debido a las mútiples terapias y presiones familiares y escolares todo aquello que significase leer era afrontado como un sobreesfuerzo excesivo.

2. Favorecer, en primer lugar, un adecuado análisis grafémico que permita asociar, a continuación, a cada grafema individual el fonema correspondiente.

3. Ensamblar los fonemas asociados en el punto anterior.

4. Facilitar el reconocimiento global de algunas palabras. 
Una vez que fuese capaz de realizar todas aquellas tareas que requieren los puntos 2 y 3 se esperaba que su lectura mejoraría sustancialmente y se pudiesen abordar otros aspectos diferentes.

En resumen, las actividades que se acordaron, conjuntamente con la profesora tutora y de apoyo, han sido las siguientes:

1. Se iba a seleccionar de forma cuidadosa que los textos fuesen del interés de Iván, que, en definitiva, serían los que se utilizasen en su propia clase.

2. Lectura en voz alta por la profesora del texto. Pondría especial cuidado en la pronunciación, articulación y entonación.

3. Lectura silenciosa, interiorizada, del texto por Iván, al igual que todos los compañeros.

4. Lectura en voz alta del texto por Iván. La profesora irá anotando los fallos que cometa.

5. Se realizará el análisis de las palabras que ha fallado en la lectura de la siguiente forma:

a) Se descompone la palabra escrita en sílabas y en letras.

b) Se atiende al sonido de las sílabas, de las letras individualmente acudiendo a la onomatopeya y a movimientos de cuerpo asociados a la onomatopeya con el objeto de ayudarle a recordar más fácilmente el sonido. Se pronuncian los sonidos individualmente.

c) Se deletrea la palabra de izquierda a derecha.

d) Se realizan varios ejercicios de composición y descomposición de la palabra (con todas las letras desordenadas se compone la palabra; a partir de la palabra se descompone y forma nuevas palabras por el cambio de una o varias letras, etc.). Estos ejercicios siempre han de estar acompañados del análisis fonológico descrito en el punto c.

e) Se escribe la palabra, se comenta su significado y se hace uso de imágenes relativas a esa palabra.

f) Análisis de palabras en sus partes correspondientes de raíz, prefijos y afijos, aprovechando los contenidos del programa escolar.

g) Se elaboran frases diversas en las que aparezcan la palabra y todas las combinaciones creadas, haciendo hincapié en el significado dentro del contexto de unas y otras.

h) Después del análisis completo de todos los fallos, se vuelve a leer el texto y se vuelven a comentar los errores.

i) Utilización de un cuaderno-guía donde se irán escribiendo las palabras que han sido objeto de análisis con su correpondiente imagen.

j) Repaso y ejercitación constante de lo trabajado.

Como se puede observar, existen algunos aspectos que no son pertinentes dentro del marco teórico en que nos desenvolvemos, pero que ha sido imposible planificar de modo diferente dados los «inconvenientes» de plantear una terapia dentro del desarrollo general de una clase de $5 .^{\circ}$ de EGB. Al objeto de salvar, en la medida de lo posible, estas dificultades, además de las generadas por la ansiedad familiar ante los problemas lectores de Iván, se ha establecido que existiese una gran coordinación entre la profesora tutora y la de apoyo con el fin de que ésta trabajase individualmente con Iván todos los aspectos señalados en el punto 5 de las actividades, a partir de las palabras que la tutora detectase en el aula que Iván leía mal.

También se puede notar que los apartados a, b, c, d, e y f del punto 5, aspectos centrales del tratamiento, intenta dotar a Iván de la adecuada capacidad de abordar las palabras por medio de las reglas de CGF (palabra escrita sílaba grafema fonema palabra hablada comprensión de la misma). 
La utilización del cuaderno-guía era una especie de diccionario a usar por Iván donde debían constar los análisis correctos de las palabras trabajadas en cuanto a su análisis grafémico.

\section{RETEST}

Nueve meses después de trabajar de esta forma se ha vuelto a pasar la lista de las palabras y pseudopalabras, obteniendo los siguientes resultados:

La lectura errónea de las palabras bajó de 42 a 5 , equivalente a un $6,94 \%$, de las que cuatro son errores visuales (Tabla IV).

Los errores en pseudopalabras descendieron desde 62 a 27 (37,5\%). También aquí se encuentran errores visuales como los de la Tabla $\mathrm{V}$.

La mejora más importante se da, como se puede observar, en la lectura de las palabras. Dado que el número de errores en las palabras es tan pequeño no se puede hacer ninguna valoración sobre el efecto de la longitud $(\mathrm{LL}=2, \mathrm{LM}=3, \mathrm{LC}=\emptyset$ ) o de la frecuencia $(F A=2, F M=2$ y FB = 1). Sin embargo, en las pseudopalabras se sigue encontrando un importante efecto de longitud ( $L L=15, L M=10, L C=2$ ), exponente claro de un uso de la ruta no léxica que, comparándola con el rendimiento que mostraba un año antes, ahora funciona muchísimo mejor.

TABLA IV

Errores visuales

\begin{tabular}{ccc}
\hline «magnífico» & $\rightarrow$ & "mognifico» \\
«decorativo" & $\rightarrow$ & "descorativo» \\
"Caracterizar" & $\rightarrow$ & "carocterizar» \\
(el otro error fue «diverso» & $\rightarrow$ & «sivero») \\
\hline
\end{tabular}

TABLA V

Errores visuales en ias pseudopalablas

\begin{tabular}{ccc}
\hline «dítulo» & $\rightarrow$ & «bitulo» \\
«escalástico» & $\rightarrow$ & "escaléntico" \\
"explosión» & $\rightarrow$ & "explesión» \\
"cremético" & $\rightarrow$ & "cremético» \\
"protafonista» & $\rightarrow$ & "protefonista" \\
"preserciar" & $\rightarrow$ & "precisar» \\
\hline
\end{tabular}

La relación NP/P aumentó a 5,4, situándose entre la que se obtiene en $3 .^{\circ}$ y en $6 .^{\circ}$, lo que es indicativo de un aumento muy importante de la ruta léxica, que es la que media en la lectura de las palabras. En la misma línea encontramos que se cometen 7 lexicalizaciones, que representa un $25,92 \%$ (equivalente al número que se realiza en $1 .^{\circ}$, un 24,91 ) del total de errores en las pseudopalabras (anteriormente era sólo un 16,25\%) y todas las comete en las pseudopalabras de LL, en línea con los resultados de los lectores normales de $1 .^{\circ}, 3^{\circ}$ y $6 .^{\circ}$. 
TABLA VI

Lexicalizaciones en el retest

\begin{tabular}{ccc}
\hline «satisfectorio" & $\rightarrow$ & "satisfactorio» \\
«cuarquiera» & $\rightarrow$ & «cualquiera» \\
«ligecamente» & $\rightarrow$ & «ligeramente» \\
«herramienta» & $\rightarrow$ & «herramienta» \\
«ensolbrecer» & $\rightarrow$ & «ensombrecer» \\
«experimontar» & $\rightarrow$ & "experimenta» \\
«experiengia» & «experiencia» \\
\hline
\end{tabular}

El efecto complementario, en este momento, de convertir las palabras en pseudopalabras muestra una disminución muy importante desde el $83,33 \%$ a un $6,94 \%$ equivalente a la que ofrecen los sujetos de $6 .^{\circ}$ curso. Esto nos hace pensar, en principio, en un dominio conjunto de la ruta directa y de las reglas de CGF.

Sigue silabeando, aunque el analisis silábico lo realiza ya de forma correcta, así como la asociación del fonema al grafema. Ahora bien, donde aún presenta cierta dificultad es al ensamblar la fonología correcta que logra. Lee correctamente las sílabas, pero al mandarle que diga la palabra completa fracasa generalmente en el intento.

La comprensión de las palabras ha mejorado sustancialmente aunque quedan pequeños restos del síntoma anterior por el que adjudicaba el significado a partir de la fonología obtenida por la ruta no léxica.

\section{Conclusión}

La conclusión a la que se puede llegar después de esta nueva valoración es que nos encontramos ante un caso más puro de dislexia fonológica que el mostrado al principio, un año antes.

El funcionamiento de la ruta léxica ha mejorado sustancialmente, como se observa en el número de errores cometidos en las palabras, en el aumento de las lexicalizaciones, en la disminución extraordinaria de los errores en las palabras en relación a las pseudopalabras y en que el significado de las palabras está mucho menos mediado por la fonología de lo que estaba un año antes.

La ruta no léxica mejoró también sustancialmente, pero aún comete un porcentaje bastante alto de errores en las pseudopalabras (37,50\%). Sin embargo, la dificultad se limita a un aspecto concreto del sistema de CGF como es el ensamblar la fonología que, en este momento, ya obtiene de forma adecuada y correcta.

\section{DISCUSION GENERAI}

El enfoque que aquí se ha dado suministra una gran esperanza a la escuela en un tema tan controvertido y complicado como es la enseñanza y la rehabilitación de los problemas de lectura. No obstante, al comenzar esta discusión general, es conveniente comenzar recordando que Iván muestra un funcionamiento deficiente del módulo de Reconocimiento de las Palabras conjuntamente con las importantes dificultades que mostraba en el conocimiento de las correspondencias grafemas a fonemas en la primera fase de la evaluación. Después del tratamiento el funcionamiento 
del léxico ortográfico se restableció sustancialmente como lo demuestra la importante mejoría que se detecta en la lectura de palabras, el aumento de lexicalizaciones y de la relación NP/P. Sin embargo, este léxico no comenzó a formarse y a ser capaz de mediar en la lectura de las palabras hasta el momento en que tanto el análisis grafémico como la asociación grafema a fonema comenzó a funcionar correctamente. Cabe aquí una pregunta relativa a la generalización de resultados desde las palabras del contexto de la clase a las que forman la prueba. ¿Cómo es posible que trabajando las palabras propias del aula, tanto fonológica como visualmente, que no son las integrantes de la prueba, se dé esa generalización de resultados? Podemos hipotetizar que al ir formándose las dos rutas de forma paralela y al unísono, el aprendizaje de las reglas grafema a fonema permita formarse, lo que Stuart y Coltheart (1988) denominan unidades de reconocimiento de la ruta léxica. Se puede interpretar que el trabajo de análisis grafémico realizado a lo largo de las palabras clave, aquellas en las que mostraba una mayor dificultad, conjuntamente con un trabajo fonológico tal como se expuso al hablar del tratamiento, pudo ir generando unidades de reconocimiento. Estas unidades, en nuestro caso, podrían ser las primeras Ietras de las palabras dado su carácter informativo (Iván, al igual que los sujetos de $1 .^{\circ}, 3 .^{\circ}$ y $6 .^{\circ}$, realiza más lexicalizaciones cuando las primeras letras de las pseudopalabras permanecen in cambiarse). Stuart y Coltheart (1988, p. 172) llegan incluso a decir:

«... sugerimos que el niño con buena capacidad de segmentación fonética y un buen conocimiento de las relaciones letra a sonido puede comenzar a construir un léxico ortográfico sin tener necesariamente alguna experiencia formal con las palabras escritas."

Esta afirmación está hecha en su sentido más fuerte, dado que es extremadamente improbable que niños de preescolar sean capaces de segmentar correctamente y de forma total todas las palabras y que efectúen una construcción ortográfica de la misma. Esta matización se reafirma al comprobar que no todas las letras se aprenden igual de fácil, pues las dependientes del contexto son más difíciles que aquellas que no lo son. Igualmente los fonemas intermedios de una palabra son más difíciles de segmentar que los iniciales y que los finales.

Aquí de momento nos limitamos a realzar la importancia del sistema de CGF en la formación del sistema de Reconocimiento de las Palabras, como también está enfatizado por el trabajo de Ehri y Wilce (1985). Se defiende, por tanto, que a patir del análisis grafémico y de la asociación de la fonología correspondiente se va formando la ruta léxica que a medida que se ejercita, por la reperición, va siendo cada vez más funcional e importante en el desarrollo lector. De no ser así, el léxico ortográfico de Iván debería funcionar en alguna medida dado el intenso tratamiento y la enorme experiencia, en tiempo y cantidad, que ha tenido con las palabras al estar en tratamiento logopédico específico desde una temprana edad. Sin embargo, cuando lo vimos por primera vez presentaba niveles inferiores a los propios de $1 .^{\circ}$ de EGB y en cuanto se trabajaron las habilidades propias de la ruta no léxica, favoreciendo con las actividades la formación de la ruta léxica, ésta comenzó a mejorar y llegó a estar, al cabo de un año, con una capacidad intermedia entre $3 .^{\circ}$ y $6 .^{\circ}$.

Lo que está claro es que Iván, a pesar de las múltiples terapias logopédicas que recibió, comenzó a «leer» cuando se inició el trabajo en esta línea, pues antes tenía una lectura equivalente a la que mostraban los alumnos de $1 .^{\circ}$ o incluso peor en algunos aspectos ya señalados. Lo cual sirve para pensar que la enorme mejoría que experimentó fue producto de un éxito, parcial todavía, del tratamiento realizado. 
Otra cuestión que cabría pensar es si el mal rendimiento en las tareas de memoria auditiva inmediata podría ser la causa de la incapacidad que muestra Iván de ensamblar la fonología. Es decir, cabe la posibilidad de que cuando llegue al final de la lectura de las palabras no se acuerde de los fonemas anteriores y, en consecuencia, tenga que inventar o decir aquello que muy vagamente recuerde, por lo que la deficiente memoria auditiva inmediata que posee sea la causa de esta dificultad. Ya se comentó en otro lugar (Lozano, 1990) que los estudios correlacionales entre Ios sujetos disléxicos y los buenos lectores dan un gran solapamiento, por lo que es posible que pueda existir una tercera variable causante de las dificultades. Asimismo, Sebastián y col. (1986) y Navalón y cols. (1989) no encontraron diferencias entre lectores normales y retrasados en tareas de memoria a corto plazo. Dado que las dificultades lectoras y la mayoría de los procesos linguísticos (entre ellos la memoria a corto plazo) son producto del funcionamiento del hemisferio cerebral izquierdo podemos concluir con Ellis (1984) que la explicación a estos datos puede estar en que tanto la dislexia como los problemas de memoria a corto plazo sean consecuencia de una tercera variable también localizada en el hemisferio cerebral izquierdo. No obstante, aún podemos decir que la razón de esta memoria deficiente puede estar más en una dificultad de saber cómo memorizar que en diferencias específicas estructurales de la memoria (Torgensen, 1977).

Otro aspecto a destacar de este estudio es el hecho que queda demostrado de la posibilidad de encontrar subtipos disléxicos en castellano, reafirmando la opinión de Masterson, Coltheart y Meara (1985) con un disléxico superficial. Además, no sólo se pueden encontrar estas dos disociaciones, sino que, dentro de la propia ruta no léxica, se comprueba que efectivamente se pueden dar tres tipos, al menos, de dificultades que son las que se han encontrado en Iván: el análisis grafémico, la asociación grafema a fonema y el ensamblaje de la fonología obtenida en un todo. Estas tres habilidades son independientes entre sí dado que pueden ir mejorando (o empeorando) individualmente como se demuestra en Iván, que al final de un año de tratamiento mejoraron las dos primeras, mientras que la capacidad de ensamblar la fonología aún no funciona tan correctamente como es de esperar.

Desde este modelo evolutivo de lectura, lo primero a determinar es el tipo de función o módulo concreto que no funciona de forma adecuada, teniendo como línea de partida el funcionamiento normal del grupo al que pertenece el sujeto (de aquí la importancia de los trabajos experimentales sobre la lectura normal). Una vez determinado este aspecto se puede planificar una terapia dirigida específicamente a él (incluso mucho más específica de lo que nosotros la hemos planificado por imponderables externos), e ir dando pequeños pasos hacia la mejora, si no total, sí al menos lo suficientemente funcional para desenvolverse tanto escolar como socialmente.

\section{Referencias}

AARON, P. G. (1989). Qualitative and quantitative differences among dyslexic, normal and non-dyslexic poor readers. Rading and Writing, 1, 291-308.

AlEgria, J. (1985). Por un enfoque psicolingüístico del aprendizaje de la lectura. Infancia y Aprendizaje, 29, 79-94.

Alegria, J.; PIGNOT, E., Y MORAIS, J. (1982). Phonetic analysis of speech and memory codes in beginning readers. Memory and Cognition, 10,451-456.

ARAM, D. M.; ROSE, D. F., Y HOR WITZ, S. J. (1982). Hyperlexia. developmental reading without meaning. En R. N. Malatesha y H. Whitaker (eds.).

BenDER, L. A. (1956). Psychology of children with organic brain disorders. Springfield: Charles C. Thomas. 
BENTON, A. L. (1971). Introducción a la Neuropsicología Ed. Fontanella. Barcelona.

Benton, A. L. (1975). Developmental dyslexia: neurological aspects. En W. J. Friedlander (ed.).

BRavo, V. L. (1981). Teorías sobre las dislexias y su enfoque cientifico. Andrés Bello. Santiago de Chile.

Bravo, V. L. (1985). Dislexias y retraso lector Enfoque netrológico. Santillana. Madrid.

BRYANT, P. Y IMPEY, L. (1986). The similarities between normal readers and developmental and acquired dyslexics. Cognition, 24, 121-137.

Caramazza, A., y McCloskey, M. (1988). The case for single patient studies. Cognitite Neuropsychology. 5, 517-528.

COLTHEART, M. (1978). Lexical access in simple reading task. En G. Underwood (ed.).

Coltheart, M. (1980). Deep dyslexia a review of the syndrome. En M. Coltheart, K. E. Patterson y J. C. Marshall (eds.).

COLTHEART, M. (1981). Disorders of reading and their implications for models of normal reading. Visible Language, XV, 245286.

COLTHEART, M. (1984). Acquired dyslexias and normal reading. En R. N. Malatesha y H. A. Whitaker (eds.).

Coltheart, M.; Patierson, K., y Marshall. J. C. (Eds.) (1980). Deep Dyslexia. Londres: Routledge \& Kegan Paul

Coltheart, M.; Patterson, K., y Marshall, J. C (Eds.) (1987). Deep Dyslexia. Londres: Routledge \& Kegan Paul, (2.a Edición).

Cuetos Vega, F. (1990). Psicología de la lectura (Diagnóstico y Tratamiento). Ed. Escuela Española, S. A. Madrid.

DuFy, F., Y GESCIWIND, N. (Eds.) (1988). Dislexia. Aspectos psicológicos y neurológicos. Labor (orig. de 1985).

EHRI, L. C., Y WILCE, L. S. (1985). Movement into reading: is the first stage of printed word learning visual or phonetic? Reading Research Quarterly, 20 (2), 163-179.

FRIEDLANDER, W. J. (Ed.) (1975). Advances neurology. Vol. 7. Nueva York: Raven Press.

FRITH, U. (ED.) (1980A). COGNTTIVE PROCESSES IN SPELLING. LONDRES: ACADEMIC PRESS.

FunNELl, E. (1983). Phonological processes in reading: nev evidence from acquired dyslexia. Brithis Journal of Psychology, 74, 159-180.

ELuS, A. W. (1984). Reading, uriting and dyslexia: a cognitive analysis. Londres: LEA.

Lozano, L. (1990). Las dificultades de la lectura desde el procesamiento de la información. Comunicación, Lenguaje y Educación. S. 105-116.

LoZANO, L. (1991). Análisis de las diferentes estrategias lectoras en los alumnos de EGB: El modelo evo lutivo de doble ruta ante el diagnóstico de las dislexias. Tesis doctoral no publicada. Univ. Oviedo.

Malatesha, R. N., Y Whitaker, H. A. (Eds.) (1984). Dyslexia: A global issue. La Haya: Martinus Nijhoff.

MARSHALL, J. C. (1984). Toward a rational taxonomy for acquired dyslexias. En R. N. Malatesha y H. A. Whitaker (eds.).

MarshaLl, J. C. (1985). A propósito de algunas relaciones entre las dislexias adquiridas y las de desarrollo. En F. H. Duffy y N. Geschwind (eds.).

MEISEL, J. (Ed.) (1986). Adquisición del lenguaje. Francfort: Verlag Klaus Dieter Veuvert.

Mitchell, D. C. (1982). The Process of Reading. A Cognitive Analysis of Fluent Reading and Learning to Read. John Wiley \& Sons Ltd. Nueva York.

MORTON, J. (1969). Interaction of information in word recognition. Psychological Review, 76. 165-178.

MORTON, J. (1980). The logogen model and orthographic structure. En U. Frith (ed.).

Morton, J., PATterson, K. E. (1980. A new attempt at an interpretation, or an attempt at new interpretation. En M. Coltheart, K. E. Pattersoh y J. C. Marshall (eds.).

Navalon, C.; ARo, M., Y RABADAN, R. (1984). El papel de la memoria de trabajo en la adquisición lectora en niños de habla castellana. Infancia y Aprendizaje .45. 85-106.

SChWARTZ, M. F.; SAFRAN, E. M., Y MARIN' O. S. M. (1980). The word order problem in agrammatism: 1, comprehension. Brain and Language, 10, 249-262.

Sesbastian, E., y Maldonado, A. (1986). Estrategias de segmentación en lectores jóvenes. En J. Meisel (ed.).

Silberberg, N., Y Silberberg, M. (1967). Hyperlexia: Secific word recognition skills in young children. Exceptional Child., 34, 41.

STUART, M. (1990). Factors influencing word recognition in pre-reading children. Britbis Joutrnal of Psychology. (en prensa).

StUart, M., Y Coltheart, M. (1988). Does reading develop in a sequence of stages? Cognition. 30, 139181

TORGENSSEN, J. K. (1977). Memorization processes in reading disabled children. Journal of Educatioral Psychology 5, 571-578.

UNDER WOOD, G. (1978). Strategies of information proccessing. Londres: Academic Press.

VALle ArroYo, F. (1991). Psicolingüística. Madrid, Morata.

Valie Arroyo, F, y Cuetos Vega, F. (1989). Las dislexias desde el enfoque neurocognitivo. San Pa, 10 9-19.

Valle Arroyo, F; Cuetos, F.; IgoA, J. M., y Viso, S. Del (Eds.) (1990). Lecturas de Psicolingïística. Vol. 1. Comprensión y producción del lenguaje. Vol. 2. Neuropsicología cognitiva del lenguaje. Madrid, Alianza. 


\section{Etended Summary}

Normal reading models viewed from an information processing perspective are opening up a positive approach to reading difficulties and their treatment. The reading model asumed in this study is the Double Route Reading Model proposed, among others, by Morton $(1969,1980)$, Coltheart $(1978,1980)$, Morton and Patterson (1980), Marshall (1984, 1985), Patterson and Shewell (1987).

The act of reading means setting into operation a series of specific components intergrated in the double route reading model. These components are independent of each orher, and have clear and specific communication links between them. Their specific role is to transform the type of information that they receive into another type.

The first part of this study briefly analyses the components which make up the two routes of the reading model. That is: a lexical route (word recognition, word comprehension, and word pronounciation); and, a phonological route (nonlexical phonological recoding, and word pronounciation).

In view of the ineffectiveness of traditional treatment observed in Ivan's case history, it was decided to treat this subject using the double route reading model. For this purpose, 144 words and nonwords were used, controlled for their length, frequency of use, and grammatical type. The relevant analyses were carried out and the results were compared with those obtained on the same tasks by students of the same chronological age or younger. On the basis of the normal reading model, suggestions are made with respect to what is happening in lvan's reading for him to make the observed mistakes. In other words, using successes and mistakes in the child's reading we hypothesize which units are functioning well, and which are not functioning or are doing so deficiently. At this point it would be possible to catalogue his difficulties in terms of some dyslexic syndrome, but this would serve no direct purpose as Ivan's difficulties might be caused by very different reasons.

Therefore, we have diagnosed that it is a case of phonological dyslexia, presenting difficulties in all three skills of grapheme-phoneme correspondences (graphemic parsing, phoneme assignment, and blending), and also in the child's visual route at the level of orthographic word recognition.

The specific therapy devised for this student concentrates on fearures relevant for reading, therefore leaving to one side traditional therapy (psychomotive, laterality, visual perception exercises, etc.). After nine months of specific treatment Ivan's reading skills were assessed once more. The results show that there has been a substantial improvement in certain areas (orthographic word recognition in the lexical route, in graphemic parsing, and phoneme-grapheme association), though there are still areas that need to improve (blending and harmonizing phonemes into a unique and coherent whole). The therapy will then concentrate on this persistent difficulty.

This way of approaching the treatment of reading difficulties shows that reading is the product of many independent skills. Indeed, the difficulties related to a unit might improve while others do not. Moreover, this approach supports the view that there are dyslexic subtypes, and that even within the same type subjects present different difficulties. Another important issue from an instructional point of view is that Ivan only improved when traditional therapies were stopped and we focussed on a therapy with an Information Processing approach. This helps to introduce fresh air both to reading instruction and to the treatment of its difficulties. 